\title{
A COMPARATIVE STUDY OF VISUAL AND PHOTOELECTRIC TIMING OF OCCULTATIONS
}

L. V. MORRISON

Royal Greenwich Obs., Herstmonceux, Sussex, England

\section{Introduction}

The timing of occultations of stars by the Moon provide data for the following studies:

(a) Lunar theory - Constants and secular changes of its orbit.

(b) Fundamental star systems - Systematic corrections to equinox and obliquity; individual star corrections.

(c) Rotation of Earth - Comparison of atomic and ephemeris time scales.

With these applications in mind we consider the analysis of the [O-C]'s from occultations.

\section{Definition of $\Delta s$}

The O-C, $\Delta s$, is defined by,

$$
\left\{225 \cos \delta \cos \delta_{*}\left(\alpha-\alpha_{*}\right)^{2}+\left(\delta-\delta_{*}\right)^{2}\right\}^{1 / 2}-(R+\varepsilon)=\Delta s
$$

where $\alpha, \delta \quad$ topocentric position of Moon $(j=2[1])$

$$
\begin{array}{ll}
\alpha_{*}, \delta_{*} & \text { apparent position of star (Robertson ZC [2]) } \\
R & \text { semi-diameter of Moon } \\
\varepsilon & \text { limb profile correction (Watts [3]). }
\end{array}
$$

\section{Errors Contributing to $\Delta s$}

\begin{tabular}{llll}
\hline$\sigma$ & & Visual & Photo-electric \\
\hline 1. Timing & Yes & No \\
2. Star place & Yes & Yes \\
3. Lunar ephemeris & Yes & Yes \\
4. Watts' limb profile & Yes & Yes \\
\hline
\end{tabular}

\section{4. $\Delta s$ for Visual Timing}

What are the magnitudes of the errors 1 to 4 and how can they be separated? The Moon's passage in front of the Pleiades of 1969 March 23 provides about 1000 observations of 22 bright stars occurring within several hours. We remove the common ephemeris error from the $\Delta s$ and plot the frequency distribution curve which is 
seen to be gaussian with a standard deviation, $\sigma_{1,2,4}=0$ ".39. We then remove most of the errors arising from star places by considering the distribution of residuals formed by taking the mean values of $\Delta s$ for each star separately. The fitted gaussian curve gives $\sigma_{1,4}=0^{\prime \prime} .29$

$$
\therefore \sigma_{2}=\left\{(0.39)^{2}-(0.29)^{2}\right\}^{1 / 2}=0.26 \text {. }
$$

This estimate agrees well with a comparison of Robertson's with Eichhorn's [4] recent photographic positions of 502 Pleiades stars for epoch 1955.0. We find,

$$
\begin{aligned}
& \sigma_{\alpha}=0.29 \\
& \sigma_{\delta}=0.23 .
\end{aligned}
$$

\section{5. $\Delta s$ for Photoelectric Timing}

About 1090 observations made at 28 different stations since 1949 were analyzed. The normal frequency distribution curve for $\Delta s$ gives $\sigma_{2,3,4}=0 " .42$. From comparisons of the lunar ephemeris with numerical integrations made by Mulholland and Devine [5] we take $\sigma_{2} \simeq 0^{\prime \prime} .2$ thus, $\sigma_{3,4}=0^{\prime \prime} 37$.

\section{Errors from Watt's Limb Chart, $\sigma_{4}$}

We have photoelectric observations of occultations of the same star made at nearly the same time from different Japanese observatories [6]. These observations sample the limb a few degrees apart and, therefore, have the same vector component in $\Delta s$ arising from errors in the star place and lunar ephemeris. The average difference of $\Delta s$ is 0 "20 from 91 such pairs of observations. This value agrees with the estimate from the analysis of graze observations [7].

\section{Timing Accuracy, $\sigma_{1}$}

Taking $\sigma_{4}=0^{\prime \prime} .20$ and the value $\sigma_{1,4}=0^{\prime \prime} .29$ for the Pleiades we find that $\sigma_{1}=0^{\prime \prime} .21$. This implies a timing accuracy of \pm 0.44 for a single observation which may be larger than expected. However, large numbers of new observers may have augmented the value.

An analysis of three ranges, each of 1000 observations, made in the period 1960-64, gives $\sigma_{1,2,3,4}=0$ " $44 \pm 0$ ".02. Comparing this with $\sigma_{2,3,4}=0$ ". 42 for photoelectric observations implies a timing accuracy of the order 0.27 .

The ratio, $\left(\frac{0.46}{0.42}\right)^{2} \simeq \frac{6}{5}$ gives the number of observations visual/photo-el to achieve the same precision of measurement. At present $n_{v} / n_{p e} \simeq 20 / 1$.

\section{Conclusions}

1. Because of errors in star places, lunar ephemeris and limb corrections, a photoelectric observation has only slightly more weight than a visual observation. 
2. With the improvement of star places, photoelectric observations will increase in weight.

3. Photoelectric observations provide a check on the systematic delay of visual timings [8], especially of reappearances.

\section{References}

[1] IAU, Report of Commission 4: 1967, Trans. Int. Astron. Union 13B, 49.

[2] Robertson, J.: 1940, Astron. Papers, Wash. X, Part II.

[3] Watts, C. B.: 1963, Astron. Papers, Wash. XVII.

[4] Eichhorn, H., Googe, W. D., Lukac, C. F. and Murphy, J. K.: 1970, Mem. Roy. Astron. Soc. 73, 125.

[5] Mulholland, J. D. and Devine, C. J.: 1968, Science 160, 874.

[6] Reports of Hydrographic Department of Japan.

[7] Morrison, L. V.: 1970, Montly Notices Roy. Astron. Soc. 149, 81.

[8] Sinzi, A. M. and Suzuki, H.: 1966, Report of Hydrographic Researches, No. 2, 75.

\section{DISCUSSION}

Dr. Wieth-Knudsen: As one of the very few (I suppose) who are using the eye-ear method for visual observations of occultations, I would encourage Mr Morrison to make an investigation (as described) of observations acquired in this way as compared to those made by a stop watch.

The increasing amount of predictions in recent years may have provided sufficient observational material of the former kind for such an investigation, and the result might encourage the use of eyeear for visual observations. 